Article

\title{
Olive Leaf Extract (OleaVita) Suppresses Inflammatory Cytokine Production and NLRP3 Inflammasomes in Human Placenta
}

\author{
Yasuaki Kaneko ${ }^{1,+}$, Michiya Sano ${ }^{1, \dagger}{ }^{+}$, Kotomi Seno ${ }^{1}$, Yuka Oogaki ${ }^{1}$, Hironori Takahashi ${ }^{2}$, \\ Akihide Ohkuchi ${ }^{2} \mathbb{D}$, Miki Yokozawa ${ }^{3}$, Ken Yamauchi ${ }^{3}$, Hisataka Iwata ${ }^{1}$, Takehito Kuwayama ${ }^{1}$ \\ and Koumei Shirasuna ${ }^{1, *}$ \\ 1 Laboratory of Animal Reproduction, Department of Animal Science, Tokyo University of Agriculture, \\ Atsugi, Kanagawa 243-0034, Japan; 43517004@nodai.ac.jp (Y.K.); 43518008@nodai.ac.jp (M.S.); \\ s11079@yahoo.co.jp (K.S.); 43519003@nodai.ac.jp (Y.O.); h1iwata@nodai.ac.jp (H.I.); \\ takehito@nodai.ac.jp (T.K.) \\ 2 Department of Obstetrics and Gynecology, Jichi Medical University, Shimotsuke, Tochigi 329-0498, Japan; \\ hironori@jichi.ac.jp (H.T.); okuchi@jichi.ac.jp (A.O.) \\ 3 Nutraceuticals Research Office, Nutrition Act Co., Ltd., Ginza, Chuo-ku, Tokyo 104-0061, Japan; \\ m-yokozawa@n-act.co.jp (M.Y.); k-yamauchi@n-act.co.jp (K.Y.) \\ * Correspondence: ks205312@nodai.ac.jp; Tel.: +81-46-270-6588; Fax: +81-46-247-4338 \\ + These authors contributed equally to this work.
}

Received: 20 March 2019; Accepted: 24 April 2019; Published: 28 April 2019

check for updates

\begin{abstract}
The placenta is essential for pregnancy and produces both pro-inflammatory and anti-inflammatory cytokines. Excessive production of inflammatory cytokines, involving interleukin-1 $\beta$ (IL-1 $\beta$ ), IL-6, and IL-8, from placental tissues is associated with pregnancy complications. Olive leaf extract has several health benefits, including anti-inflammatory functions. OleaVita is a new commercial olive leaf extract; it is hypothesized to suppress placental inflammation. In human placental tissue culture, OleaVita treatment inhibited the secretion of inflammatory cytokines and NF-KB p65 protein expression. OleaVita also suppressed toll-like receptor ligands-induced IL-1 $\beta$ secretion in human placental tissues. IL-1 $\beta$ is regulated by the NLRP3 inflammasomes, a pivotal regulator of various diseases. OleaVita significantly decreased NLRP3 and pro-IL-1 $\beta$ protein expression, suggesting that it has an inhibitory effect on NLRP3 inflammasome activation. Thus, OleaVita is beneficial as an inhibitor of inflammation and NLRP3 inflammasome activation, and may be used as a supplement for the treatment and prevention of inflammatory diseases.
\end{abstract}

Keywords: placenta; interleukin; NLRP3 inflammasome; inflammation

\section{Introduction}

The placenta is an important organ for pregnancy, and produces both pro-inflammatory and anti-inflammatory cytokines, which regulate placental function and development [1]. Compared with the non-pregnant state, normal pregnancy is associated with increased systemic inflammation [2]. Alternatively, women with pregnancy complications, such as preterm, gestational diabetes, intrauterine growth restriction, and preeclampsia [3,4] develop accelerated systemic and placental inflammation [5]. Levels of pro-inflammatory cytokines such as interleukin (IL)-1 $\beta$, IL-6, and IL-8 have been observed in the sera and placental tissues of pregnant women with these complications compared with those in healthy pregnant women [2,5-7]. However, because of concerns of toxicity to the fetus, pregnancy complications are very difficult to treat with drugs. 
Inflammatory responses associated with gestational hypertension and preeclampsia can occur even in the absence of microbial infection. Thus, these syndromes are considered to be sterile inflammations [8,9]. Sterile inflammatory responses are mediated through nucleotide-binding oligomerization domain-like receptor (NLR) family pyrin domain-containing 3 (NLRP3) inflammasomes. NLRP3 inflammasomes are large multi-protein complexes in the cytosol that regulate the production of the pivotal inflammatory cytokine IL-1 $\beta[10,11]$. NLRP3 inflammasomes are comprised of three different proteins: NLRP3; apoptosis-associated speck-like protein containing a caspase recruitment domain (ASC); and caspase-1 (an IL-1 $\beta$-converting enzyme). NLRP3 inflammasomes are associated with various types of inflammatory diseases, such gout, cardiovascular diseases, metabolic syndrome, type 2 diabetes, and silicosis [10-13]. In the placenta and peripheral blood leukocytes of preeclamptic patients, the expression of NLRP3, caspase-1, and IL-1 $\beta$ is higher than in those of healthy pregnant women [14]. Pregnancy complications induced by angiotensin II, nanosilica, and extracellular vesicles are improved in NLRP3-deficient mice since the suppression of placental inflammation $[9,15,16]$. These findings suggest that the inhibition of NLRP3 inflammasomes is essential to counter pregnancy dysfunctions.

The olive, Olea europaea, grows in the Mediterranean regions, Asia, and Africa, and is one of the most relevant traditional drugs. Olive leaf extracts is known to be a natural resource of various beneficial substances, such as polyphenols, oleuropein, and oleanolic acid, and have been used for various effects, including anti-inflammatory, anti-hypertensive, and hypocholesterolemic activities [17,18]. There are many reports stating that oleanolic acid has an anti-inflammatory effect both in vivo and in vitro [19-22]. Recently, a new product, an ethanol/water extract of olive leaves (OleaVita ${ }^{\circledR}$, Phytodia S.A.S., Illkirch-Graffenstaden, France), has been developed [23]. This extract contains large amounts of oleanolic acid, which activates TGR5 (G protein-coupled bile acid receptor 1) and is reported to have an obesity-suppressing effect in humans [23]. However, to the best of our knowledge, there have been no investigations into the effect of olive leaf extracts, including OleaVita, on human placental function.

In the present study, we assess the role of the olive leaf extract OleaVita in suppressing inflammatory cytokine production and in NLRP3 inflammasome activation in human placenta. To test our hypothesis, we investigated whether OleaVita has an inflammatory inhibitory effect and what mechanism controls inflammation using in vitro human placental tissue culture model.

\section{Materials and Methods}

\subsection{Olive Leaf Extract (OleaVita)}

OleaVita ${ }^{\circledR}$, an ethanol/water extract of olive leaves, was used in the present study. It was obtained from Phytodia S.A.S. (Illkirch-Graffenstaden, France). A powdered extract of OleaVita was dissolved in dimethylsulfoxide (DMSO) at $2 \mathrm{~g} / \mathrm{mL}$ as a stock solution.

\subsection{Tissue Collection}

Human placentae were obtained from a total of twelve women, who delivered healthy, singleton infants at term (patients who gave informed consent were obtained using protocols approved by the Jichi Medical University Ethics Committee). The relevant clinical details of the patients are shown in Table 1 . In the present study, we used 4-5 placentas in each experiment and totally we used 12 placentas (9 placenta of females and 3 placenta of males). As shown in our previous study [24], tissues were obtained within $20 \mathrm{~min}$ of delivery and dissected fragments were placed in ice-cold phosphate buffered saline (PBS). Placental tissue was blunt dissected to remove visible connective tissue and cut into small pieces (about $50 \mathrm{mg}$ wet weight). These placental tissue pieces were placed on a cell culture insert membrane (0.4 $\mu \mathrm{m}$ membrane, 24-well plate, Thermo Fisher Scientific, Inc., Waltham, MA, USA) with 1 mL Dulbecco's modified Eagle's medium/F-12 (DMEM/F-12; Life Technologies Corporation, Carlsbad, CA, USA) supplemented with antibiotics including amphotericin B and gentamicin (Sigma-Aldrich, St. Louis, MO, USA), and 5\% fetal calf serum (FCS, ICN Pharmaceuticals, Inc., Costa Mesa, CA, USA). 
Table 1. Individual characteristics.

\begin{tabular}{cc}
\hline Variable & Normal Pregnancy $(\boldsymbol{n}=\mathbf{1 2})$ \\
\hline Age of mother & $35.4(27-40)$ \\
Maximum systolic blood pressure $(\mathrm{mmHg})$ & $112.3(88-132)$ \\
Maximum diastolic blood pressure $(\mathrm{mmHg})$ & $70.8(48-96)$ \\
Delivery gestation (week) & $38.0(37-39)$ \\
Placental weight $(\mathrm{g})$ & $540.3(415-627)$ \\
Birthweight $(\mathrm{g})$ & $2712.7(2066-3660)$ \\
\hline
\end{tabular}

Data represented as median (range).

\subsection{Experimental Conditions}

Experiment 1: Human placental tissues were incubated with control or OleaVita $(0.1 \mathrm{or} 1 \mathrm{mg} / \mathrm{mL})$ for $6 \mathrm{~h}$ at $37^{\circ} \mathrm{C}$. In preliminary experiment, we checked the appreciate dose of OleaVita $(0.01,0.1,1$, or $2 \mathrm{mg} / \mathrm{mL}$ ) and incubation time ( 6 or $24 \mathrm{~h}$ ). Supernatant and tissues were collected for enzyme-linked immunosorbent assay (ELISA), western blotting, and real-time Reverse transcription polymerase chain reaction (RT-PCR), and were stored at $-20^{\circ} \mathrm{C}$ or $-80^{\circ} \mathrm{C}$ before use.

Experiment 2: To investigate the role of Jun N-terminal kinase (JNK), human placental tissues were incubated with OleaVita $(1 \mathrm{mg} / \mathrm{mL}$ ) with or without SP600125 (a JNK inhibitor, $10 \mu \mathrm{M}$, Merck Millipore, Burlington, MA, USA), for $6 \mathrm{~h}$ at $37^{\circ} \mathrm{C}$. Supernatant and tissues were collected for ELISA and were stored at $-20^{\circ} \mathrm{C}$ before use.

Experiment 3: To investigate the role of OleaVita on toll-like receptor (TLR) ligand-induced inflammatory responses, human placental tissues were pre-incubated with or without OleaVita $(1 \mathrm{mg} / \mathrm{mL}$ ) for $1 \mathrm{~h}$. Tissues were then incubated with zymosan (TLR2 agonist, $50 \mu \mathrm{M}$, Invivogen, Carlsbad, CA, USA), lipopolysaccharide (LPS, TLR4 agonist, $1 \mu \mathrm{g} / \mathrm{mL}$, Sigma-Aldrich), or imiquimod (TLR7 agonist, $20 \mu \mathrm{M}$, Invivogen) for $6 \mathrm{~h}$ at $37^{\circ} \mathrm{C}$. Supernatant and tissues were collected for ELISA and were stored at $-20^{\circ} \mathrm{C}$ before use.

\subsection{Determination of Cytokines}

Levels of IL-1 $\beta$, IL-6, or IL-8 were determined using a human ELISA kit (R\&D Systems, Minneapolis, MN, USA) according to the manufacturer's instructions.

\subsection{Real-Time RT-PCR}

Total RNA was prepared using ISOGEN II (Nippon Gene Company, Limited, Toyama, Japan) according to the manufacturer's instructions. RNA extraction and cDNA production were performed using a commercial kit (ReverTra Ace; Toyobo Co., Ltd., Osaka, Japan). Real-time RT-PCR was performed using the CFX Connect ${ }^{\mathrm{TM}}$ Real Time PCR (Bio-Rad, Hercules, CA, USA) and a commercial kit (Thunderbird SYBR qPCR Mix; Toyobo Co., Ltd.) to detect mRNA expressions of IL-1 $\beta$, IL-6, IL-8, Caspase-3, or glyceraldehyde 3-phosphate dehydrogenase (GAPDH). The following antisense and sense primers were used: $I L-1 \beta\left(5^{\prime}\right.$ - TGATGGCTTATTACAGTGGCAATG- $3^{\prime}$ and $5^{\prime}-$ GTAGTGGTGGTGGGAGATTCG-3' ${ }^{\prime}$ NM-000576), IL-6 (5' - AAATTCGGTACATCCTCGACGG $-3^{\prime}$ and 5'- GGAAGGTTCAGGTTGTTTTCTGC-3', M54894), IL-8 (5'- CTTGGCAGCCTTCCTGATTTC $-3^{\prime}$ and $5^{\prime}-$ GGTGGAAAGGTTTGGAGTATGTCT $-3^{\prime}$, BC013615.1), Caspase-3 (5'CATGCAAGCGAATCAATGGACT $-3^{\prime}$ and $5^{\prime}$ - CTGTACCAGACCGAGATGTCA $-3^{\prime}$, AJ413269.1), and GAPDH (5' - AAATGAGCCCCAGCCTTCT-3' and 5' - AGGATGTCAGCGGGAGCCGG-3', M33197). RT-qPCR was performed in duplicate with a final reaction volume of $20 \mu \mathrm{L}$ containing $10 \mu \mathrm{L}$ SYBR Green, $7.8 \mu \mathrm{L}$ distilled water, $0.1 \mu \mathrm{L} 100 \mu \mathrm{M}$ forward and reverse primers, and $2 \mu \mathrm{L}$ of cDNA template. The amplification program consisted of a 5 min denaturation at $95{ }^{\circ} \mathrm{C}$ followed by 40 cycles of amplification $\left(95^{\circ} \mathrm{C}\right.$ for $15 \mathrm{~s}, 60^{\circ} \mathrm{C}$ for $30 \mathrm{~s}$, and $72{ }^{\circ} \mathrm{C}$ for $20 \mathrm{~s}$ ). Expression levels of each target gene were normalized to corresponding GAPDH threshold cycle (CT) values using the $\Delta \Delta \mathrm{CT}$ comparative method [25]. 


\subsection{Determination of Cytokines and Lactate Dehydrogenase (LDH)}

After incubation, supernatant were collected and stored at $-20{ }^{\circ} \mathrm{C}$ before use. Levels of LDH as a cell death marker was determined using cytotoxicity detection kit (Roche Diagnostics $\mathrm{GmbH}$, Mannheim, Germany).

\subsection{Western Blot Analysis}

Lysates from the placental tissues were prepared using RIPA buffer (Wako Pure Chemical Industries, Osaka, Japan). Placental tissues were washed with cold PBS, minced and incubated with RIPA buffer for $15 \mathrm{~min}$ on ice into $1.5 \mathrm{~mL}$ tubes. Placental tissue lysates were subsequently centrifuged at $12,000 \times g$ for $20 \mathrm{~min}$ at $4{ }^{\circ} \mathrm{C}$. Supernatants were transferred to a fresh tube and stored at $-80{ }^{\circ} \mathrm{C}$ before analysis. A total of $10 \mu \mathrm{g}$ protein was loaded per lane and separated by $10 \%$ SDS-PAGE. The expression of protein was analyzed by western blot. After transfer onto polyvinylidene fluoride membranes, nonspecific antibody binding was blocked for $1 \mathrm{~h}$ at room temperature using Immunoblock (DS Pharma Biomedical Co, Ltd, Osaka, Japan). Then, membranes were incubated for $24 \mathrm{~h}$ at $4{ }^{\circ} \mathrm{C}$ with anti-NF-кB p65 antibody (1:500, EMD Millipore Corporation, Temecula, CA, USA), anti-phospho-IкB antibody (1:500, R\&D systems), anti-IKB antibody (1:500, R\&D systems), anti-IL-1 $\beta$ antibody (1:1000, Santa Cruz Biotechnology, Dallas, TX, USA), anti-NLRP3 antibody (1:250, R\&D systems), anti-ASC antibody (1:1000, Santa Cruz Biotechnology, Santa Cruz, CA, USA), anti-phospho-c-Jun antibody (1:1000, Cell Signaling Technology, Danvers, MA, USA), anti-c-Jun antibody (1:1000, Cell Signaling Technology), and anti- $\beta$-actin (ACTB) antibody (1:10000, Sigma-Aldrich), followed by an incubation for $1 \mathrm{~h}$ with secondary antibody conjugated horseradish peroxidase (HRP; 1:1000, GE Healthcare, UK Ltd, Buckinghamshire, UK). Immunoreactive bands were visualized by Western BLoT Quant HRP Substrate (GE Healthcare) using ImageQuant LAS 4000 (GE Healthcare). The results represent at least 3 independent experiments. Quantitative analysis of bands was performed using Image J (National Institutes of Health, Bethesda, MD, USA).

\subsection{Statistical Analysis}

Data are expressed as mean \pm standard error of the mean (SEM). Differences between treatment groups were identified nonparametric analysis of variance, followed by Mann-Whitney U-test or Kruskal test. A $p$-value of $<0.05$ was considered statistically significant.

\section{Results}

\subsection{Effects of OleaVita on Inflammatory Cytokine Production in Human Placental Tissues}

We examined the effects of OleaVita on the regulation of inflammatory cytokine production using human placental tissue cultures. The secretion of the cytokines IL-1 $\beta$, IL-6, and IL-8 in human placental tissue culture were significantly decreased following OleaVita treatment (Figure 1A-C). Similar with cytokine secretions, the expression of the corresponding mRNAs was also decreased after OleaVita treatment (Figure 1D-F). On the other hand, treatment with OleaVita did not affect the levels of LDH (as a cell death marker) and mRNA expression of caspase-3 (as an apoptosis marker), suggesting that OleaVita has no toxic effects on human placenta (Figure 1G,H). In this experiment, we used three placentas of females and two placentas of males and results indicated that the response to OleaVita did not change between placentas from females and males (Figure S1).

\subsection{Effects of OleaVita on the NF- $\kappa B$ Pathway in Human Placental Tissues}

It is well known that NF- $\kappa \mathrm{B}$ is a key transcription factor for the production of inflammatory cytokines [26]. Transcriptional activity of NF- $\kappa B$ is dependent on I $\kappa B$ phosphorylation as an NF- $\kappa B$ inhibitor. After I $\mathrm{kB}$ phosphorylation, the NF- $\mathrm{BB}$ p65 subunit is transported into the nucleus, where it promotes the expression of inflammatory cytokines. Although there was no observed effect on 
phospho-IкB and total-IкB protein expression, treatment with OleaVita considerably reduced NF- $\mathrm{kB}$ p65 protein expression in human placental tissue culture (Figure 2A-D).

(A) IL-1 $\beta$ secretion

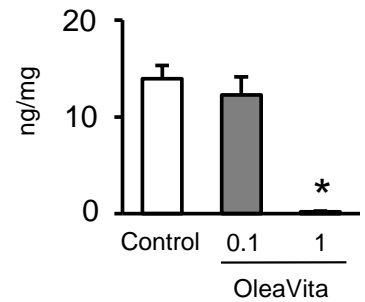

(D) IL-1 $\beta$ mRNA

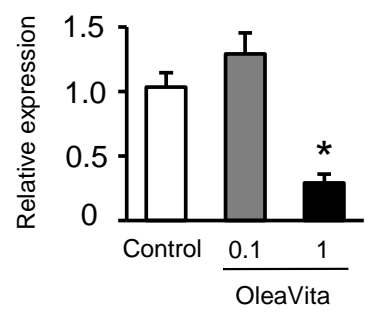

(G) $\mathrm{LDH}$ release

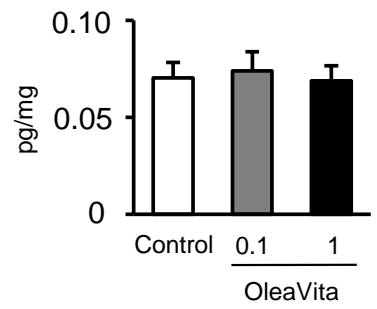

(B) IL-6 secretion

(C) IL-8 secretion

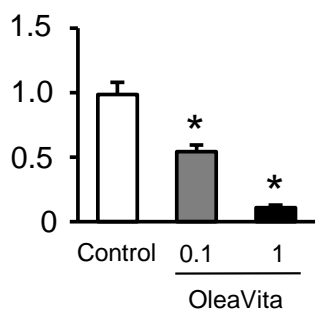

(E) IL-6 mRNA

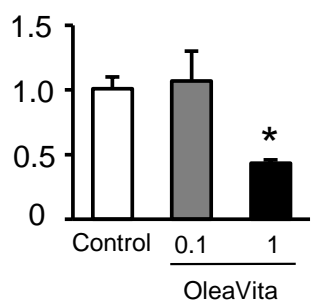

(H) Caspase-3 mRNA

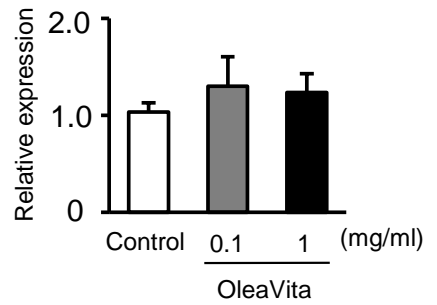

Figure 1. Effects of OleaVita on inflammatory cytokine production in human placental tissues. Human placental tissues were incubated for $6 \mathrm{~h}$ with OleaVita $(0.1$ or $1 \mathrm{mg} / \mathrm{mL})$. After incubation, IL-1 $\beta$ (A), IL-6 (B), and IL-8 (C) levels in the supernatant were determined using ELISA. The IL-1 $\beta$, NLRP3, ASC, CASP1, and Caspase-3 mRNA levels were measured using qRT-PCR (D-F, and $\mathbf{H})$. The release of LDH levels were subsequently quantified in the supernatant $(\mathbf{G})$. Data are expressed as means $\pm \operatorname{SEM}(n=5$, 3 female and 2 male placentas). Differences between treatment groups were identified nonparametric analysis of variance, followed by a Kruskal test; ${ }^{*} p<0.05$.

(A) Placental protein expression

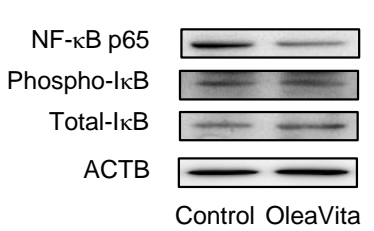

(B) NF-кB p65

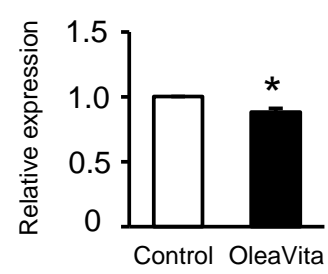

(C) Phospho-IkB

(D) Total-IкB

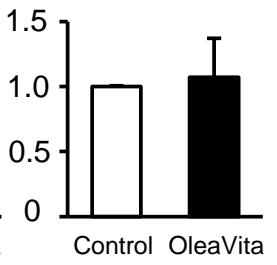

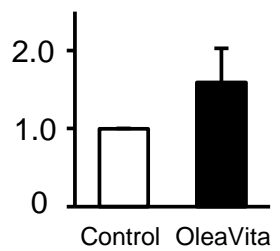

Figure 2. Effects of OleaVita on the NF- $\mathrm{KB}$ pathway in human placental tissues. Human placental tissues were incubated for $6 \mathrm{~h}$ with OleaVita $(1 \mathrm{mg} / \mathrm{mL})$. After incubation, NF-кB p65, phospho-IкB, total-IKB, and ACTB protein levels were subsequently quantified in the cell lysates by western blot (A-D). Data are expressed as means $\pm \operatorname{SEM}(n=4,2$ female and 2 male placentas). Differences between treatment groups were identified nonparametric analysis of variance, followed by the Mann-Whitney $U$-test; ${ }^{*} p<0.05$. 


\subsection{Effects of OleaVita on NLRP3 Inflammasomes in Human Placental Tissues}

Because OleaVita clearly suppressed IL-1 $\beta$ secretion from human placental tissue, we assessed the potential role of OleaVita in inhibiting NLRP3 inflammasome activation. As expected, OleaVita treatment significantly decreased pro-IL-1 $\beta$ and NLRP3 protein expression in human placental tissues (Figure 3A-C). OleaVita treatment significantly decreased the expression of mRNA for pro-IL-1 $\beta$ and NLRP3 in human placental tissues [27]. Conversely, treatment with OleaVita did not affect ASC protein expression (Figure 3A). These findings suggest that OleaVita inhibits mature IL-1 $\beta$ secretion via the down-regulation of both pro-IL-1 $\beta$ and NLRP3 inflammasomes in human placenta.

(A) Placental protein expression

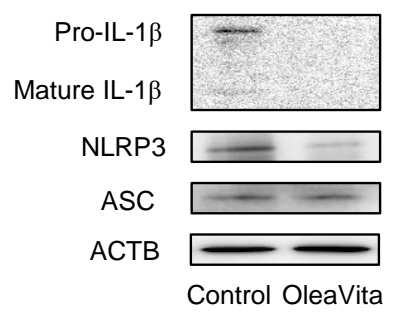

(B) pro-IL-1 $\beta$

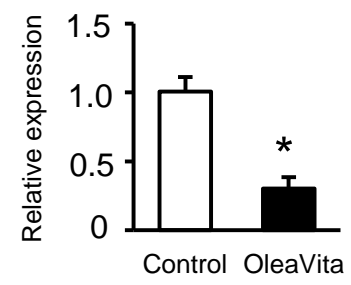

(C) NLRP3

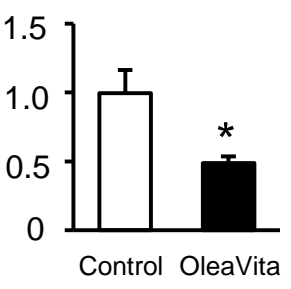

Figure 3. Effects of OleaVita on NLRP3 inflammasomes in human placental tissues. Human placental tissues were incubated for $6 \mathrm{~h}$ with OleaVita $(1 \mathrm{mg} / \mathrm{mL})$. After incubation, IL-1 $\beta$, NLRP3, ASC, and ACTB protein levels were subsequently quantified in the cell lysates by western blot $(\mathrm{A}-\mathrm{C})$. Data are expressed as means \pm SEM $(n=4,2$ female and 2 male placentas). Differences between treatment groups were identified nonparametric analysis of variance, followed by the Mann-Whitney $U$-test; $* p<0.05$.

\subsection{Effects of OleaVita on the JNK Pathway in Human Placental Tissues}

It has been reported that oleanolic acid, one of the major components of Oleavita, activates the c-Jun-N-terminal kinase (JNK) pathway, resulting in the regulation of inflammatory cytokines [28]. JNK also phosphorylates and activates the transcriptional factor c-Jun, one of the major molecules involved in JNK signaling. Therefore, we investigated the role of the JNK pathway in mediating the effects of OleaVita in human placenta. OleaVita treatment remarkably increased the expression of phosphorylated c-Jun protein, but not of total c-Jun protein, in human placental tissues (Figure 4A-C). To determine the importance of the JNK pathway, placental tissues were incubated with JNK inhibitor (SP600125) before treatment with OleaVita. Although treatment with OleaVita clearly decreased the secretion of IL-1 $\beta$ and IL-6, JNK inhibitor considerably attenuated the inflammation suppressing effect of OleaVita (Figure 4D,E). These findings indicate that the activation of the JNK pathway is necessary for the inhibitory effect of OleaVita on inflammatory cytokine production in human placenta.

\subsection{Effects of OleaVita on TLR Ligands-Induced IL-1 $\beta$ Secretion in Human Placental Tissues}

Finally, we investigated whether excessive inflammation of the TLR ligand signal can be reduced by OleaVita, and whether there is selectivity in the anti-inflammatory effects of OleaVita in human placenta. In our placental tissue culture model, three TLR ligands, including zymosan (TLR2), LPS (TLR4), and imiquimod (TLR7), remarkably stimulated IL-1 $\beta$ secretion in human placental tissues (Figure 5A-C). However, two TLR ligands, poly(I:C) (TLR3) and CPG ODN (TLR9), did not stimulate IL-1 $\beta$ secretion (data not shown). Importantly, treatment with OleaVita dramatically suppressed the secretion of IL-1 $\beta$ induced by the three TLR ligands in human placental tissues (Figure 5A-C). Thus, our findings indicated that OleaVita has a role in suppressing excessive inflammatory responses, independent of TLR ligands, in human placenta. 


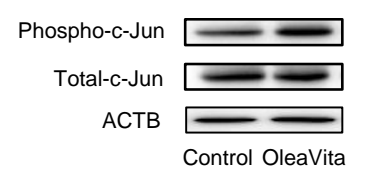

(D) IL-1 $\beta$ secretion

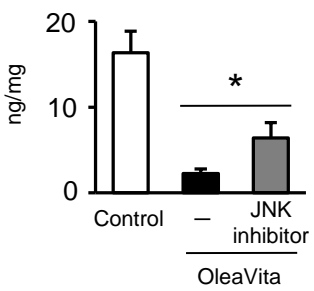

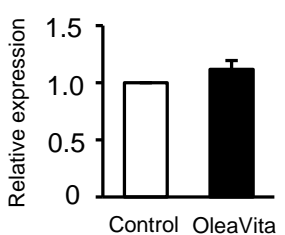

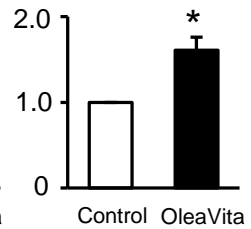

(E) IL-6 secretion

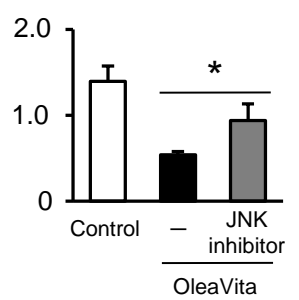

Figure 4. Effects of OleaVita on the JNK pathway in human placental tissues. Human placental tissues were incubated for $6 \mathrm{~h}$ with OleaVita $(1 \mathrm{mg} / \mathrm{mL})$. After incubation, phosphor-c-Jun, c-Jun, and ACTB protein levels were subsequently quantified in the cell lysates by western blot (A-C). Tissues were pre-incubated for $1 \mathrm{~h}$ with specific pharmacological inhibitors (SP600125 as a JNK inhibitor). After inhibitor treatments, tissues were incubated for $6 \mathrm{~h}$ with Oleavita $(1 \mathrm{mg} / \mathrm{mL})$. IL- $1 \beta$ and IL-6 levels in the supernatant were determined using ELISA (D and E). Data are expressed as means $\pm \operatorname{SEM}(n=4$, 3 female and 1 male placentas). Differences between treatment groups were identified nonparametric analysis of variance, followed by the Mann-Whitney $U$-test; ${ }^{*} p<0.05$.

\section{IL-1 $\beta$ secretion}

(A) TLR2 activation

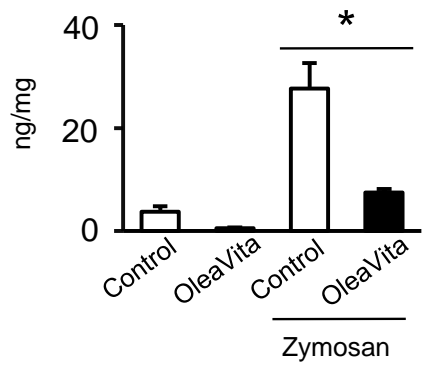

(B) TLR4 activation

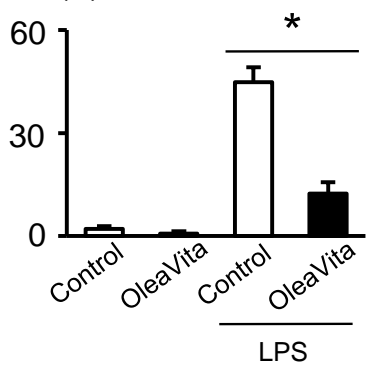

(C) TLR7 activation

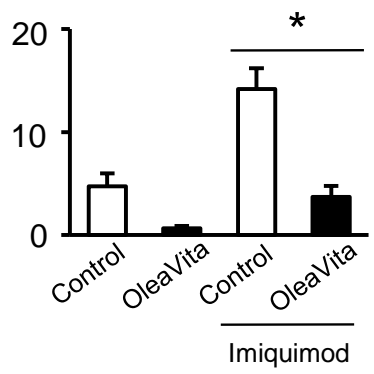

Figure 5. Effects of OleaVita on TLR ligands-induced IL-1 $\beta$ secretion in human placental tissues. Human placental tissues were pre-incubated for $1 \mathrm{~h}$ with OleaVita $(1 \mathrm{mg} / \mathrm{mL})$. After incubation, tissues were incubated for $6 \mathrm{~h}$ with each TLR-ligands (zymosan as TLR2 ligand, LPS as TLR4 ligand, and Imiquimod as TLR7 ligand). After inhibitor treatments, IL-1 $\beta$ levels in the supernatant were determined using ELISA (A-C). Data are expressed as means $\pm \operatorname{SEM}(n=4$, all female placentas). Differences between treatment groups were identified nonparametric analysis of variance, followed by the Mann-Whitney U-test; ${ }^{*} p<0.05$.

\section{Discussion}

Increasing evidence indicates that olive leaf extracts have various beneficial effects, such as the inhibition of inflammation, reduction of oxidative stress, and amelioration of apoptosis in the kidney, liver, aorta, and intestine $[17,18]$. However, the role of olive leaf extract, including OleaVita, in human placental tissues has not been understood until now. In the present study, we showed that OleaVita treatment directly inhibited the secretion of inflammatory cytokines, including IL-1 $\beta$, IL-6, and IL-8, with a decrease in the expression of their mRNA via suppression of the NF- $\mathrm{B}$ transcription pathway in human placental tissues. OleaVita has already been used in humans (It is a standard of about $1 \mathrm{~g}$ daily for supplements) and any toxic effects have not been noted (No toxicity after continuous 
administration of $2 \mathrm{~g} / \mathrm{kg} /$ day for 28 days in rats). Therefore, similar to other olive leaf extracts, OleaVita has the potential to be used as a supplement for the prevention and treatment of inflammatory diseases, involving pregnancy complications, in humans. Before applying to human pregnancy, it is necessary to examine the safety of experimental animals during pregnancy.

OleaVita has been found to have an effect which is agonistic to that of TGR5 [23]. It has been reported that overexpression of TGR5 in THP-1 cells (a human monocyte cell line) decreases the production of LPS-stimulated cytokine [29]. Oleanolic acid, one of the major components of OleaVita, is known to be a TGR5 agonist, and oleanolic acid activates JNK via TGR5, resulting in the regulation of inflammatory cytokine production and a reduction in obesity [28,30]. In the present study, treatment with OleaVita activated c-Jun downstream from the JNK pathway, and treatment with a JNK inhibitor attenuated the inflammation suppressing effect of OleaVita. Therefore, it is considered that the inflammation suppressing effect of OleaVita may be mediated through the TGR5-JNK-NF- $\kappa$ B pathway in human placenta.

The NLRP3 inflammasomes have emerged as a key regulator of various types of chronic and noninfectious inflammatory responses and diseases [10,11]. Importantly, we demonstrated that in human placental tissues, OleaVita notably inhibited the activation of NLRP3 inflammasomes, resulting in a decrease in the secretion of mature IL-1 $\beta$. Consistent with our present results, An et al. [31] and Kim et al. [19] reported that oleanolic acid attenuated carotid artery and pulmonary injuries through the inhibition of NLRP3 inflammasomes using in vivo experimental models. Recently, NLRP3 inflammasome activation has been shown to be involved in various pregnancy complications, such as preeclampsia, spontaneous preterm labor, and hypertension in pregnancy [9,14]. Thus, OleaVita, which has potential as a supplementation for the inhibition of NLRP3 inflammasomes, may lead to the alleviation of various types of pregnancy complications and non-communicative disorders in humans. In the future, it will be important to examine how OleaVita exerts its effects in specific disease models and human beings.

Because the OleaVita used in the present study is an extract of olive leaves and includes a range of components, it is difficult to identify the components responsible for the effect of OleaVita. Among various these components, oleanolic acid is one of the main components of OleaVita [23]; and oleanolic acid has been found to have strong anti-inflammatory effect and improves pathological condition. For example, oleanolic acid treatment has been shown to alleviate lung injury in mice, including the inhibition of immune cell accumulation and inflammatory cytokine production [19]. Djeziri et al. [20] reported that oleanolic acid exerts protective effects on obesity, including a decrease in body and adipose tissue weight and inflammatory cytokine production. In the reproductive experimental field, Zhao et al. [32] showed that oleanolic acid effectively rejuvenated testicular function by reducing DNA damage, apoptosis, and inflammatory cytokine production, indicating a potential for the prevention of aging-related testicular dysfunction. On the other hand, as OleaVita contains many other components, such as oleuropein and polyphenolic compounds, further studies are necessary warranted to understand the exacts effect of OleaVita.

Mitochondria are one of the targets of olive leaf extracts such as OleaVita. Indeed, OleaVita treatment increased mRNA expression of PGC1 $\alpha$, which promotes the synthesis of mitochondria in 3T3-L1 adipocytes [23]. In addition, oleanolic acid mitigates the dysfunction of mitochondrial membrane potential and ATP production induced by high glucose levels in chondrocytes [22]. In the present study, treatment with OleaVita increased mRNA expression of PGC1 $\alpha$ and factors regulating mitochondrial fusion (mitofusion (MFN) 1 and 2 and optic atrophy 1 (OPA1)) and division (dynamin-related protein 1 (DRP1)) in human placental tissues (Figure S2), suggesting a potential role for OleaVita in the regulation of mitochondrial biogenesis. However, there was no effect on mitochondrial copy number, probably because of the short incubation time $(6 \mathrm{~h})$ used in the present study. To determine the effect of OleaVita on mitochondrial function, further investigation is needed, using placental cells rather than placental tissues. 


\section{Conclusions}

In conclusion, we demonstrated that exposure to OleaVita results in the suppression of inflammatory cytokine production and the activation of NLRP3 inflammasomes, and TLR-ligands induced inflammatory responses in human placenta. In addition, JNK/NF- $\kappa \mathrm{B}$ signaling plays a pivotal role in the inhibitory effect of OleaVita on inflammatory responses. Further insights into the exact role of OleaVita in obesity, placental inflammation, and pregnancy complications will be useful for developing preventive and therapeutic strategies for these complications.

Supplementary Materials: The following are available online at http://www.mdpi.com/2072-6643/11/5/970/s1, Figure S1: Effects of OleaVita on IL-1 $\beta$ secretion depending on the sex in human placental tissues, Figure S2: Effects of OleaVita on mitochondria in human placental tissues.

Author Contributions: Conceptualization, K.S.; Investigation, Y.K., M.S., K.S., and Y.O.; Resources, H.T., M.Y., and K.Y.; Writing-Original Draft Preparation, Y.K., K.S.; Writing-Review\&Editing, A.O., H.I., T.K.; Funding Acquisition, K.S.

Funding: This study received grants from the Japan Society for the Promotion of Science (JSPS) through the Scientific Research (C) (K.S.).

Acknowledgments: The authors would like to thank Enago (www.enago.jp) for the English language review. This manuscript was reviewed by a professional service (KUSR-25, Enago Inc., Valley Cottage, NY, USA) prior to submission.

Conflicts of Interest: The authors declare no conflict of interest.

\section{Abbreviations}

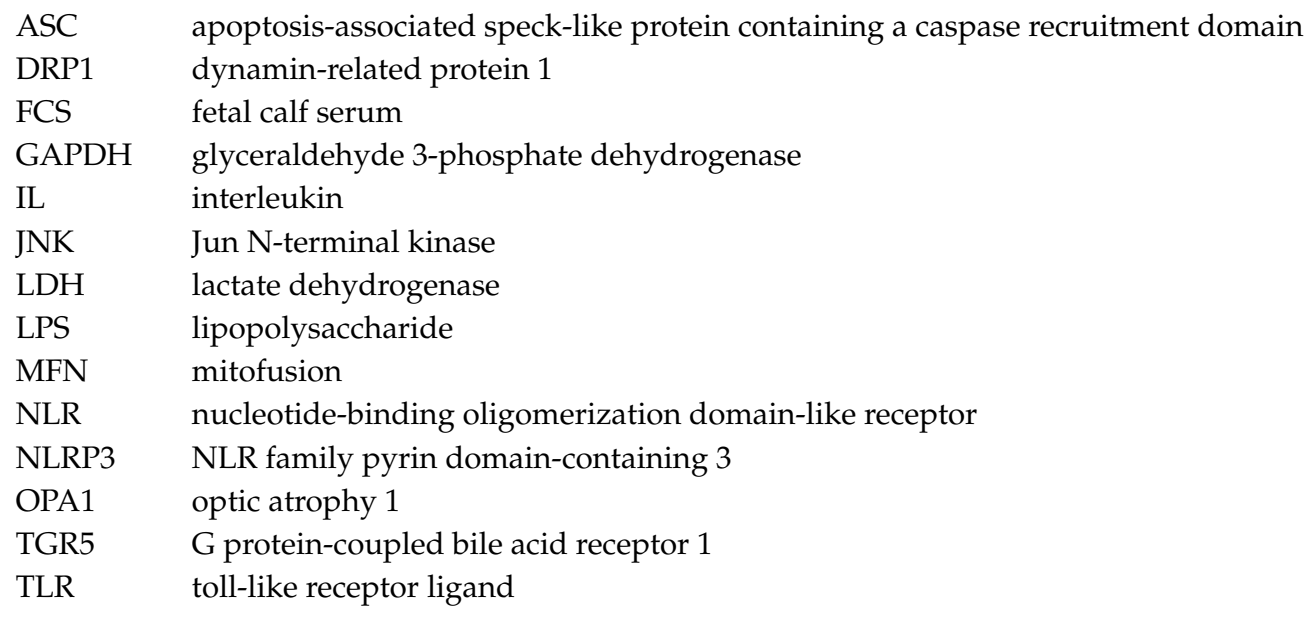

\section{References}

1. Arck, P.C.; Hecher, K. Fetomaternal immune cross-talk and its consequences for maternal and offspring's health. Nat. Med. 2013, 19, 548-556. [CrossRef]

2. Aye, I.L.; Jansson, T.; Powell, T.L. Interleukin-1beta inhibits insulin signaling and prevents insulin-stimulated system A amino acid transport in primary human trophoblasts. Mol. Cell Endocrinol. 2013, 381, 46-55. [CrossRef] [PubMed]

3. Marchi, J.; Berg, M.; Dencker, A.; Olander, E.K.; Begley, C. Risks associated with obesity in pregnancy, for the mother and baby: A systematic review of reviews. Obes. Rev. 2015, 16, 621-638. [CrossRef]

4. Catalano, P.M.; Ehrenberg, H.M. The short- and long-term implications of maternal obesity on the mother and her offspring. BJOG 2006, 113, 1126-1133. [CrossRef]

5. Basu, S.; Haghiac, M.; Surace, P.; Challier, J.C.; Guerre-Millo, M.; Singh, K.; Waters, T.; Minium, J.; Presley, L.; Catalano, P.M.; et al. Pregravid obesity associates with increased maternal endotoxemia and metabolic inflammation. Obesity (Silver Spring) 2011, 19, 476-482. [CrossRef] 
6. Challier, J.C.; Basu, S.; Bintein, T.; Minium, J.; Hotmire, K.; Catalano, P.M.; Hauguel-de Mouzon, S. Obesity in pregnancy stimulates macrophage accumulation and inflammation in the placenta. Placenta 2008, 29, 274-281. [CrossRef] [PubMed]

7. $\quad$ Roberts, K.A.; Riley, S.C.; Reynolds, R.M.; Barr, S.; Evans, M.; Statham, A.; Hor, K.; Jabbour, H.N.; Norman, J.E.; Denison, F.C. Placental structure and inflammation in pregnancies associated with obesity. Placenta 2011, 32, 247-254. [CrossRef] [PubMed]

8. Laresgoiti-Servitje, E. A leading role for the immune system in the pathophysiology of preeclampsia. J. Leukoc. Biol. 2013, 94, 247-257. [CrossRef]

9. Shirasuna, K.; Karasawa, T.; Usui, F.; Kobayashi, M.; Komada, T.; Kimura, H.; Kawashima, A.; Ohkuchi, A.; Taniguchi, S.; Takahashi, M. NLRP3 Deficiency Improves Angiotensin II-Induced Hypertension But Not Fetal Growth Restriction During Pregnancy. Endocrinology 2015, 156, 4281-4292. [CrossRef]

10. Davis, B.K.; Wen, H.; Ting, J.P. The inflammasome NLRs in immunity, inflammation, and associated diseases. Annu. Rev. Immunol. 2011, 29, 707-735. [CrossRef] [PubMed]

11. Takahashi, M. Role of the inflammasome in myocardial infarction. Trends Cardiovasc. Med. 2011, $21,37-41$. [CrossRef]

12. Schroder, K.; Zhou, R.; Tschopp, J. The NLRP3 inflammasome: A sensor for metabolic danger? Science 2010, 327, 296-300. [CrossRef] [PubMed]

13. Chen, G.Y.; Nunez, G. Sterile inflammation: sensing and reacting to damage. Nat. Rev. Immunol. 2010, 10, 826-837. [CrossRef] [PubMed]

14. Weel, I.C.; Romao-Veiga, M.; Matias, M.L.; Fioratti, E.G.; Peracoli, J.C.; Borges, V.T.; Araujo, J.P., Jr.; Peracoli, M.T. Increased expression of NLRP3 inflammasome in placentas from pregnant women with severe preeclampsia. J. Reprod. Immunol. 2017, 123, 40-47. [CrossRef] [PubMed]

15. Shirasuna, K.; Usui, F.; Karasawa, T.; Kimura, H.; Kawashima, A.; Mizukami, H.; Ohkuchi, A.; Nishimura, S.; Sagara, J.; Noda, T.; et al. Nanosilica-induced placental inflammation and pregnancy complications: Different roles of the inflammasome components NLRP3 and ASC. Nanotoxicology 2015, 9, 554-567. [CrossRef] [PubMed]

16. Shirasuna, K.; Takano, H.; Seno, K.; Ohtsu, A.; Karasawa, T.; Takahashi, M.; Ohkuchi, A.; Suzuki, H.; Matsubara, S.; Iwata, H.; Kuwayama, T. Palmitic acid induces interleukin-1beta secretion via NLRP3 inflammasomes and inflammatory responses through ROS production in human placental cells. J. Reprod. Immunol. 2016, 116, 104-112. [CrossRef]

17. Efentakis, P.; Iliodromitis, E.K.; Mikros, E.; Papachristodoulou, A.; Dagres, N.; Skaltsounis, A.L.; Andreadou, I. Effects of the olive tree leaf constituents on myocardial oxidative damage and atherosclerosis. Planta. Med. 2015, 81, 648-654. [CrossRef] [PubMed]

18. Elgebaly, H.A.; Mosa, N.M.; Allach, M.; El-Massry, K.F.; El-Ghorab, A.H.; Al Hroob, A.M.; Mahmoud, A.M. Olive oil and leaf extract prevent fluoxetine-induced hepatotoxicity by attenuating oxidative stress, inflammation and apoptosis. Biomed Pharmacother 2018, 98, 446-453. [CrossRef]

19. Kim, M.S.; Han, J.Y.; Kim, S.H.; Jeon, D.; Kim, H.Y.; Lee, S.W.; Rho, M.C.; Lee, K. Oleanolic acid acetate attenuates polyhexamethylene guanidine phosphate-induced pulmonary inflammation and fibrosis in mice. Respir. Physiol. Neurobiol. 2018, 252, 1-9. [CrossRef]

20. Djeziri, F.Z.; Belarbi, M.; Murtaza, B.; Hichami, A.; Benammar, C.; Khan, N.A. Oleanolic acid improves diet-induced obesity by modulating fat preference and inflammation in mice. Biochimie 2018, 152, 110-120. [CrossRef]

21. Martin, R.; Cordova, C.; San Roman, J.A.; Gutierrez, B.; Cachofeiro, V.; Nieto, M.L. Oleanolic acid modulates the immune-inflammatory response in mice with experimental autoimmune myocarditis and protects from cardiac injury. Therapeutic implications for the human disease. J. Mol. Cell Cardiol. 2014, 72, 250-262. [CrossRef] [PubMed]

22. Kang, X.; Yang, Z.; Sheng, J.; Liu, J.B.; Xie, Q.Y.; Zheng, W.; Chen, K. Oleanolic acid prevents cartilage degeneration in diabetic mice via PPARgamma associated mitochondrial stabilization. Biochem. Biophys. Res. Commun. 2017, 490, 834-840. [CrossRef] [PubMed]

23. Takayanagi, S.; Suzuki, H.; Yokozawa, M.; Uchiyama, K.; Ishikawa, M. The effect of the intake of ethanol/water extract of olive leaves (EEO) on body fat percentage and lean body mass percentage: A randomized, double-blind, placebo-controlled, parallel-group study of healthy japanese people. Pharmacometrics 2016, 91, 115-121. 
24. Seno, K.; Sase, S.; Ozeki, A.; Takahashi, H.; Ohkuchi, A.; Suzuki, H.; Matsubara, S.; Iwata, H.; Kuwayama, T.; Shirasuna, K. Advanced glycation end products regulate interleukin-1beta production in human placenta. J. Reprod. Dev. 2017, 63, 401-408. [CrossRef] [PubMed]

25. Livak, K.J.; Schmittgen, T.D. Analysis of relative gene expression data using real-time quantitative PCR and the 2(-Delta Delta C(T)) Method. Methods 2001, 25, 402-408. [CrossRef] [PubMed]

26. Brasier, A.R. The nuclear factor-kappaB-interleukin-6 signalling pathway mediating vascular inflammation. Cardiovasc. Res. 2010, 86, 211-218. [CrossRef]

27. Kaneko, Y.; Tokyo University of Agriculture, Kanagawa, Japan. Unpublished work. 2019.

28. Lou, G.; Ma, X.; Fu, X.; Meng, Z.; Zhang, W.; Wang, Y.D.; Van Ness, C.; Yu, D.; Xu, R.; Huang, W. GPBAR1/TGR5 mediates bile acid-induced cytokine expression in murine Kupffer cells. PLoS ONE 2014, 9 , e93567. [CrossRef] [PubMed]

29. Kawamata, Y.; Fujii, R.; Hosoya, M.; Harada, M.; Yoshida, H.; Miwa, M.; Fukusumi, S.; Habata, Y.; Itoh, T.; Shintani, Y.; et al. A G protein-coupled receptor responsive to bile acids. J. Biol. Chem. 2003, 278, 9435-9440. [CrossRef]

30. Sato, H.; Genet, C.; Strehle, A.; Thomas, C.; Lobstein, A.; Wagner, A.; Mioskowski, C.; Auwerx, J.; Saladin, R. Anti-hyperglycemic activity of a TGR5 agonist isolated from Olea europaea. Biochem. Biophys. Res. Commun. 2007, 362, 793-798. [CrossRef]

31. An, Q.; Hu, Q.; Wang, B.; Cui, W.; Wu, F.; Ding, Y. Oleanolic acid alleviates diabetic rat carotid artery injury through the inhibition of NLRP3 inflammasome signaling pathways. Mol. Med. Rep. 2017, 16, 8413-8419. [CrossRef]

32. Zhao, H.; Liu, J.; Song, L.; Liu, Z.; Han, G.; Yuan, D.; Wang, T.; Dun, Y.; Zhou, Z.; Liu, Z. Oleanolic acid rejuvenates testicular function through attenuating germ cell DNA damage and apoptosis via deactivation of NF-kappaB, p53 and p38 signalling pathways. J. Pharm. Pharmacol. 2017, 69, 295-304. [CrossRef]

(C) 2019 by the authors. Licensee MDPI, Basel, Switzerland. This article is an open access article distributed under the terms and conditions of the Creative Commons Attribution (CC BY) license (http://creativecommons.org/licenses/by/4.0/). 